\title{
Strategy-Proof Location Functions on Finite Graphs
}

\author{
F.R. McMorris \\ Department of Applied Mathematics \\ Illinois Institute of Technology \\ Chicago, IL 60616 USA \\ and \\ Department of Mathematics \\ University of Louisville \\ Louisville, KY 40292 USA \\ e-mail: mcmorris@iit.edu \\ Henry Martyn Mulder \\ Econometrisch Instituut \\ Erasmus Universiteit \\ P.O. Box 1738, 3000 DR Rotterdam, The Netherlands \\ e-mail: hmmulder@few.eur.nl \\ Fred S. Roberts \\ DIMACS Center \\ Rutgers University \\ Piscataway, NJ 08854 USA \\ e-mail: froberts@dimacs.rutgers.edu
}

February 25, 2013 
Acknowledgements Fred Roberts thanks the National Science Foundation for support under grant SES-1024722 to Rutgers University and the Department of Homeland Security for support under award 2009-ST-061-CCI002-04 to Rutgers University.

Dedication This paper is dedicated to our friend and colleague Boris Mirkin on the occasion of his 70th birthday.

\begin{abstract}
A location function on a finite graph takes a set of most preferred locations (vertices of the graph) for a set of users, and returns a set of locations satisfying conditions meant to please the entire user set as much as possible. A strategyproof location function is one for which it never benefits a user to report a suboptimal preferred location. We introduce four versions of strategy-proof and prove some preliminary results focusing on two well-known location functions, the median and the center.
\end{abstract}

\title{
1 Introduction
}

A common problem to many location studies is to find a location or set of locations that satisfy a group of customers in a way that is as good as possible, usually by maximizing or minimizing various optimization criteria. The customers are often viewed as "voters" where each one reports a preferred location on a graph, and the location function returns a set of "winners." Most of the work done in this area focuses on developing algorithms to find these optimal location vertices, but in recent years, there have been axiomatic studies of the procedures themselves. This is the approach we take in this note. We seek to understand those location functions that encourage voters/customers to report their true location preferences. That is, no voter $j$ should be able to improve the outcome (from $j$ 's point-of-view) by reporting a suboptimal location in their vote. Standard terminology labels these functions as being "strategy-proof", and the literature on this topic is extensive. For example see [13] for the many references therein. Our goal is to develop the notion of strategyproofness as it pertains to the vertex set of a finite graph with added graph-theoretic structure. We deviate from many studies (e.g. see $[1,12]$ ) by requiring all locations and customers to be on vertices of the graph, and that the edges have no real-valued lengths assigned to them. We introduce four precise concepts of strategy-proofness in our context and give some preliminary results about them. Specifically, we illustrate the concepts by looking at two well-known location functions, the median and the center, and we study these functions on several classes of graphs.

\section{Preliminaries and an elementary result}

Throughout we let $G=(V, E)$ be a finite, connected graph without loops or multiple edges, with vertex set $V$ and edge set $E$. The distance $d(u, v)$ between two 
vertices $u$ and $v$ of $G$ is the length of a shortest $u, v$-path, so that $(V, d)$ is a finite metric space. If $X \subseteq V$ and $v \in V$, then we set $d(v, X)=\min \{d(v, x): x \in X\}$. Let $k$ be a positive integer. Sequences in $V^{k}$ are called profiles and a generic one is denoted $\pi=\left(x_{1}, \ldots, x_{k}\right)$. Let $\{\pi\}$ be the set of distinct vertices appearing in $\pi$ and $|\pi|$ be number of elements in $\{\pi\}$. By $\pi\left[x_{j} \rightarrow w\right]$ we denote the profile obtained from $\pi=\left(x_{1}, \ldots, x_{j}, \ldots, x_{k}\right)$ by replacing $x_{j}$ by $w$. So $\pi\left[x_{j} \rightarrow w\right]=$ $\left(x_{1}, \ldots, x_{j-1}, w, x_{j+1}, \ldots, x_{k}\right)$, for $1<j<k$, and $\pi\left[x_{1} \rightarrow w\right]=\left(w, x_{2}, \ldots, x_{k}\right)$, and $\pi\left[x_{k} \rightarrow w\right]=\left(x_{1}, \ldots, x_{k-1}, w\right)$.

Without any conditions imposed, a location function ( of order $k$ ) on $G$ is simply a mapping $L_{V}: V^{k} \rightarrow 2^{V} \backslash\{\emptyset\}$, where $2^{V}$ denotes the set of all subsets of $V$. When the set $V$ is clear from the context, we will write $L$ instead of $L_{V}$. A single-valued location function on $G$ is a function of the form $L: V^{k} \rightarrow V$. (Notice that a single-valued $L$ can be viewed as requiring $|L(\pi)|=1$ for all $\pi$.) Given a profile $\pi$, we can think of $x_{i}$ as the reported location desired by customer (or voter) $i$, and $L(\pi)$ as the set of locations produced by the function $L$. To measure how "close" a vertex $x$ is to a given profile $\pi=\left(x_{1}, \ldots, x_{k}\right)$, the values of $s(x, \pi)=\sum_{i=1}^{k} d\left(x, x_{i}\right)$ and $e(x, \pi)=\max \left\{d\left(x, x_{1}\right), \ldots, d\left(x, x_{k}\right)\right\}$ have been often used. We will be concerned with two well-studied location functions (e.g., see $[4,5,6]$ ) which return vertices close, in the previous sense, to a given profile. The center function is the location function Cen $: V^{k} \rightarrow 2^{V} \backslash\{\emptyset\}$ defined by $\operatorname{Cen}(\pi)=\{x \in V: e(x, \pi)$ is minimum $\}$. The median function is the location function $M e d: V^{k} \rightarrow 2^{V} \backslash\{\emptyset\}$ defined by $\operatorname{Med}(\pi)=\{x \in V$ : $s(x, \pi)$ is minimum $\}$.

A single-valued $L$ is onto if, for any vertex $v$ of $G$, there exists a profile $\pi$ such that $L(\pi)=v$. A location function $L$ is unanimous if, for each constant profile $(u, u, \ldots, u)$ on $u$ consisting only of occurrences of the vertex $u$, we have $L((u, u, \ldots, u))=\{u\}$.

The interpetation of a profile $\left(x_{1}, x_{2}, \ldots, x_{k}\right)$ is that $x_{j}$ represents the most preferred location for voter $j$. Assuming that voter $j$ wants to have the decision rule or location function lead to a choice of $x_{j}$ or at least to include $x_{j}$ in the set of chosen alternatives, how can a decision rule or location function prevent $j$ from misrepresenting his or her true preference in order to gain an advantage. This is the intuitive notion of strategy-proofness and the following is an attempt to make this precise for location functions. Let $L: V^{k} \rightarrow 2^{V} \backslash\{\emptyset\}$ be a location function of order $k$ on $G$. Then $L$ is strategy-proof of the type $S P i$ if, for $i \in\{1,2,3,4\}, L$ satisfies the following:

SP1: For every profile $\pi=\left(x_{1}, \ldots, x_{k}\right) \in V^{k}, j \in\{1, \ldots, k\}$ and $w \in V$,

$$
d\left(x_{j}, L(\pi)\right) \leq d\left(x_{j}, L\left(\pi\left[x_{j} \rightarrow w\right]\right)\right) .
$$

SP2: For every profile $\pi=\left(x_{1}, \ldots, x_{k}\right) \in V^{k}$ and $j \in\{1, \ldots, k\}$, if $x_{j} \notin L(\pi)$, then there does not exist a $w \in V$ such that $x_{j} \in L\left(\pi\left[x_{j} \rightarrow w\right]\right)$.

SP3: For every profile $\pi=\left(x_{1}, \ldots, x_{k}\right) \in V^{k}$, if $x_{j} \in L(\pi)$ with $|L(\pi)|>1$, then there does not exist a $w \in V$ such that $\left\{x_{j}\right\}=L\left(\pi\left[x_{j} \rightarrow w\right]\right)$. 
SP4: For every profile $\pi=\left(x_{1}, \ldots, x_{k}\right) \in V^{k}$ and $j \in\{1, \ldots, k\}$, if $x_{j} \notin L(\pi)$, then there does not exist a $w \in V, w \neq x_{j}$, such that $\left\{x_{j}\right\}=L\left(\pi\left[x_{j} \rightarrow w\right]\right)$.

Clearly SP1 implies SP2 implies SP4.

\section{Examples}

1. SP2 does not imply SP1: This example draws on ideas found in [11]. Let $G$ be the path on three vertices denoted in order $a_{1}, a_{2}, a_{3}$, and let $L(\pi)=a_{j}$ where $a_{j} \in\{\pi\}$ appears most frequently in $\pi$ and $j$ is the smallest index among such vertices. Now let $\pi=\left(x_{1}, x_{2}, x_{3}\right)=\left(a_{1}, a_{2}, a_{3}\right)$. Then $L(\pi)=a_{1}$ and $d\left(x_{3}, L(\pi)\right)=2$. But $d\left(x_{3}, L\left(\pi\left[x_{3} \rightarrow a_{2}\right]\right)=d\left(a_{3}, L\left(a_{1}, a_{2}, a_{2}\right)\right)=d\left(a_{3}, a_{2}\right)=1\right.$.

2. SP4 does not imply SP2: We will show in Proposition 6 that $C e n$ is such an example on the path on four vertices.

If $L$ is single-valued then SP3 does not apply and SP2 and SP4 are equivalent. Also, when $L$ is single-valued, SP1 corresponds to the definition found in [12]: voter $j$ will never be able to improve (from her/his point-of-view) the result of applying the location function by reporting anything other than their peak choice $x_{j}$. SP2 implies that if voter $j$ 's top choice is not returned by $L$, then it cannot be made a part of the output set by $j$ 's reporting something else as top choice. SP3 requires that when $j$ 's top choice is returned by $L$ along with others, this choice cannot be made into the unique element in the output set by reporting something else. Finally, SP4 says that when $j$ 's top choice is not returned by $L$, it cannot be the unique output returned by $L$ if $j$ reports a different choice.

The following result appears to be well-known ([2], [12]) but we include a proof for completeness since our context differs, as mentioned previously.

Lemma 1 Let $L$ be a single-valued location function of order $k$ on $G$ that satisfies $S P 1$. Then $L$ is onto if and only if $L$ is unanimous.

Proof. Clearly, a unanimous location function is onto.

Conversely assume that $L$ is onto and let $u$ be an arbitrary vertex of $G$. Because $L$ is onto, there is a profile $\pi=\left(y_{1}, y_{2}, \ldots, y_{k}\right)$ with $L(\pi)=u$. Let $\rho=\left(x_{1}, x_{2}, \ldots, x_{k}\right)$ be the profile with $x_{j}=u$ for all $j$, and let $\pi_{0}=\rho$. For $j=1,2, \ldots, k$, let $\pi_{j}=\pi_{j-1}\left[x_{j} \rightarrow y_{j}\right]$. Note that $\pi_{k}=\pi$. Since $L$ satisfies SP1, we have

$$
d\left(u, L\left(\pi_{j-1}\right)\right) \leq d\left(u, L\left(\pi_{j}\right)\right),
$$

for $j=1,2, \ldots, k$. Hence

$$
d(u, L(\rho)) \leq d(u, L(\pi))=0,
$$

and the proof is complete. 


\section{$3 \quad$ Strategy-proof functions on paths}

We first consider the simplest situation: the graph is a path. This corresponds to the problem of locating a vertex along a single highway, or street, and is a fairly standard case to be considered ([7],[8]). Let $P$ be a path of length $n$. Without loss of generality we may assume that $V=\{0,1, \ldots, n\}$ is the vertex set of $P$ with the vertices on $P$ numbered consecutively so that $P=0 \rightarrow 1 \rightarrow \ldots \rightarrow n$. Note that $d(u, v)=|u-v|$ for $u, v \in V$.

We now consider single-valued location functions of order $k$ on $P$.

Let $G$ be the graph $P^{k}$, that is, the Cartesian product of $k$ copies of $P$. Thus $V^{k}$ is the vertex set of $G$, and two vertices $\pi=\left(x_{1}, \ldots, x_{k}\right)$ and $\rho=\left(y_{1}, \ldots, y_{k}\right)$ of $G$ are adjacent if and only if there is exactly one $i$ such that $\left|x_{i}-y_{i}\right|=1$, and $x_{j}=y_{j}$ for all $j \neq i$. The distance function on $G$ is given by

$$
d(\pi, \rho)=\sum_{i=1}^{k}\left|x_{i}-y_{i}\right|
$$

where $\pi=\left(x_{1}, \ldots, x_{k}\right)$ and $\rho=\left(y_{1}, \ldots, y_{k}\right)$ are vertices of $G$.

Clearly $V$ is a linearly ordered set under $\leq$, the usual ordering on the natural numbers. This can be used to induce a partial ordering, which we also denote by $\leq$, on $V^{k}$ as follows: for $\pi=\left(x_{1}, \ldots, x_{k}\right)$ and $\rho=\left(y_{1}, \ldots, y_{k}\right)$ in $V^{k}$ define

$$
\pi \leq \rho \text { if and only if } x_{i} \leq y_{i} \text { for all } 0 \leq i \leq k
$$

We denote the poset $\left(V^{k}, \leq\right)$ by $G_{\leq}$. Note that $\rho=\left(y_{1}, \ldots, y_{k}\right)$ covers $\pi=$ $\left(x_{1}, \ldots, x_{k}\right)$ in $G_{\leq}$if, for some $i$, we have $y_{i}-x_{i}=1$ with $x_{j}=y_{j}$ for all $j \neq i$. Because we want to focus on the graph structure as well as the order, we use $G_{\leq}$in the sequel.

A location function $L: V^{k} \rightarrow V$ is isotone on $G_{\leq}$if, for any two vertices $\pi$ and $\rho$ of $G_{\leq}, \pi \leq \rho$ implies $L(\pi) \leq L(\rho)$.

Theorem 2 Let $L$ be a single-valued location function of order $k$ on the path $P$ of length $n$ and let $G=P^{k}$. If $L$ satisfies $S P 1$, then $L$ is isotone on $G_{\leq}$.

Proof. First we prove that $L$ is order preserving on each edge of the Hasse diagram of $G_{\leq}$. Let $\pi \rho$ be an edge in $G$ with $\pi=\left(x_{1}, \ldots, x_{j-1}, x_{j}, x_{j+1}, \ldots, x_{k}\right)$ and $\rho=\left(x_{1}, \ldots, x_{j-1}, x_{j}+1, x_{j+1}, \ldots, x_{k}\right)$. Thus $\rho$ covers $\pi$ in $G_{\leq}$. We have to prove that $L(\pi) \leq L(\rho)$. For convenience we write $x=x_{j}$ and $x_{j}^{\prime}=x_{j}+1$.

Assume to the contrary that $L(\pi)>L(\rho)$. We consider three cases.

Case 1. $L(\pi)>L(\rho) \geq x+1$. 
Note that we can write $\rho=\pi\left[x_{j} \rightarrow x+1\right]$. Since $L$ satisfies SP1, this implies that

$$
d\left(x_{j}, L(\pi)\right) \leq d\left(x_{j}, L\left(\pi\left[x_{j} \rightarrow x+1\right]\right),\right.
$$

which can be written as

$$
d(x, L(\pi)) \leq d(x, L(\rho)) .
$$

Due to the choice of $V$ and the distance function $d$ of $P$, this amounts to

$$
L(\pi)-x \leq L(\rho)-x,
$$

which is impossible.

Case 2. $x \geq L(\pi)>L(\rho)$.

Note that we can write $\pi=\rho\left[x_{j}^{\prime} \rightarrow x\right]$. SP1 implies that

$$
d\left(x_{j}^{\prime}, L(\rho)\right) \leq d\left(x_{j}^{\prime}, L\left(\rho\left[x_{j}^{\prime} \rightarrow x\right]\right),\right.
$$

which can be written as

$$
d(x+1, L(\rho)) \leq d(x+1, L(\pi)) .
$$

Due to the properties of the distance function $d$ on $P$, this amounts to

$$
x+1-L(\rho)) \leq x+1-L(\pi),
$$

which is impossible.

Case 3. $L(\pi) \geq x+1>x \geq L(\rho)$.

Note that we can write $\rho=\pi\left[x_{j} \rightarrow x+1\right]$. Then SP1 implies that

$$
d\left(x_{j}, L(\pi)\right) \leq d\left(x_{j}, L\left(\pi\left[x_{j} \rightarrow x+1\right]\right)\right),
$$

which can be written as

$$
d(x, L(\pi)) \leq d(x, L(\rho)) .
$$

Due to the properties of the distance function $d$ on $P$ this amounts to

$$
L(\pi)-x \leq x-L(\rho) .
$$

Hence we have

$$
L(\pi)+L(\rho) \leq 2 x .
$$

Now we write $\pi=\rho\left[x_{j}^{\prime} \rightarrow x\right]$. Then SP1 gives that

$$
d\left(x_{j}^{\prime}, L(\rho) \leq d\left(x_{j}^{\prime}, L\left(\rho\left[x_{j}^{\prime} \rightarrow x\right]\right),\right.\right.
$$

which can be written as

$$
d(x+1, L(\rho)) \leq d(x+1, L(\pi)) .
$$


This amounts to

$$
x+1-L(\rho) \leq L(\pi)-(x+1) .
$$

Hence we have

$$
2(x+1) \leq L(\pi)+L(\rho) .
$$

Clearly (1) and (2) yield a contradiction, which proves that $L$ preserves order on the edges of $G_{\leq}$.

Now consider any two vertices $\pi$ and $\rho$ of $G_{\leq}$with $\pi \leq \rho$. Since $L$ is isotone on edges of $G_{\leq}$, it is isotone on all the edges in a shortest ordered path from $\pi$ to $\rho$, which implies that $L(\pi) \leq L(\rho)$.

The converse of Theorem 2 is not true, even if the isotone location function is onto.

Example Define the average function $A$ on $P$ by $A(\pi)=\left\lfloor\frac{1}{k} \sum_{i=1}^{k} x_{i}\right\rfloor$, where $\pi=$ $\left(x_{1}, \ldots, x_{k}\right)$. It is straightforward to check that the average function is an isotone, onto location function on $G_{\leq}$, but that it does not satisfy SP1. For a specific example, consider $\pi=\left(x_{1}, \ldots, x_{k}\right)=(0,1, \ldots, 1,1)$ and $\pi\left[x_{k} \rightarrow 2\right]$. Then $A(\pi)=(k-1) / k$ and $A\left(\pi\left[x_{k} \rightarrow 2\right]\right)=1$ so $d\left(x_{k}, A(\pi)\right)>d\left(x_{k}, A\left(\pi\left[x_{k} \rightarrow 2\right]\right)\right.$.

Theorem 3 Let $L$ be an onto single-valued location function on the path $P$ of length $n$ that satisfies SP1. Then

$$
\min _{x_{j} \in \pi}\left(x_{j}\right) \leq L(\pi) \leq \max _{x_{j} \in \pi}\left(x_{j}\right)
$$

for any profile $\pi$ on $P$.

Proof. Set $\alpha=\min _{x_{j} \in \pi}\left(x_{j}\right)$ and $\beta=\max _{x_{j} \in \pi}\left(x_{j}\right)$. By Lemma 1, we have $L((\alpha, \alpha, \ldots, \alpha))=\alpha$ and $L((\beta, \beta, \ldots, \beta))=\beta$. Then in $G_{\leq}$there is an ordered path from $(\alpha, \alpha, \ldots, \alpha)$ to $(\beta, \beta, \ldots, \beta)$ passing through $\pi$. Since $L$ satisfies SP1, the assertion now follows from Theorem 2 .

\section{Strategy-Proofness of the Center Function}

In this section we investigate how $C e n$ behaves on paths, complete graphs, cycles, and graphs with diameter greater than 2 . Let $P_{n}$ denote the path $a_{1} a_{2} \cdots a_{n}$ with $n$ vertices, and let $K_{n}$ denote the complete graph on $n$ vertices. Recall that the diameter of a graph $G$ is the maximum $d(x, y)$ for $x, y \in V$. Since $C e n$ is unanimous, trivially Cen satisfies SP1, SP2, SP3, and SP4 on $P_{1}=K_{1}$.

Proposition 4 Let $G=K_{n}$ and $k>1$. Then Cen satisfies SP1, SP2, SP3, SP4 on $G$. 
Proof. If $\pi=\left(x_{1}, \ldots, x_{k}\right)$ is a profile with $|\pi|=1$, we are done since $C e n$ is unanimous. So assume $|\pi|>1$. Then $\operatorname{Cen}(\pi)=V$ and $\operatorname{Cen}\left(\pi\left[x_{j} \rightarrow w\right]\right)=V$ or $\operatorname{Cen}\left(\pi\left[x_{j} \rightarrow w\right]\right)=\{w\}$. SP1 holds since $d\left(x_{j}, V\right)=0$, and therefore SP2 and SP4 hold. SP3 holds because if $\left|\operatorname{Cen}\left(\pi\left[x_{j} \rightarrow w\right]\right)\right|=1$, then $\operatorname{Cen}\left(\pi\left[x_{j} \rightarrow w\right) \neq\left\{x_{j}\right\}\right.$.

Proposition 5 Let graph $G$ have diameter at least 3 and $k>1$. Then Cen violates conditions SP1 and SP2.

Proof. Let $a u_{1} u_{2} \cdots u_{p}$ be a shortest path of length at least 3 from $a$ to $u_{p}$, so $p \geq 3$. Let $\pi=\left(x_{1}, \ldots, x_{k}\right)=\left(a, a, \ldots, a, u_{2}\right)$. Then $\operatorname{Cen}(\pi)=\left\{v \in V: a v \in E, u_{2} v \in E\right\}$. Now $\operatorname{Cen}\left(\left(\pi\left[x_{k} \rightarrow u_{3}\right]\right)=\operatorname{Cen}\left(\left\{a, a, \ldots, u_{3}\right\}\right)\right.$ contains $u_{1}$ and $u_{2}$. In particular, $x_{k}=u_{2} \in \operatorname{Cen}\left(\left(\pi\left[x_{k} \rightarrow u_{3}\right]\right)\right.$ while $x_{k} \notin \operatorname{Cen}(\pi)$. Thus, SP2 fails and therefore so does SP1.

\subsection{Paths}

We now consider the center function on the path $P_{n}$ of $n$ vertices, which we will denote in order on the path as $a_{1} a_{2} \cdots a_{n}$. We may consider $n>2$ since $n=2$ gives us a complete graph and so here SP1 through SP4 hold by Proposition 4.

Proposition 6 Suppose Cen is defined on $P_{n}$ for $n>2$, and let $k>1$. Then

1. Cen satisfies SP1 if and only if $n=3$.

2. Cen satisfies SP2 if and only if $n=3$.

3. Cen fails SP3 for all $n>2$.

4. Cen satisfies SP4 if and only if $n \in\{3,4\}$.

Proof. We first observe that SP3 fails for $n>2$. If $\pi=\left(x_{1}, \ldots, x_{k}\right)=\left(a_{1}, a_{1}, \ldots, a_{1}, a_{2}\right)$, then $\operatorname{Cen}(\pi)=\left\{a_{1}, a_{2}\right\}$. However, $\operatorname{Cen}\left(\pi\left[x_{k} \rightarrow a_{3}\right]\right)=\operatorname{Cen}\left(\left(a_{1}, a_{1}, \ldots, a_{1}, a_{3}\right)\right)=$ $\left\{a_{2}\right\}$, which contradicts condition SP3.

We next consider SP1, SP2, and SP4 for the case $n=3$. It suffices to show that SP1 holds, for then SP2 and SP4 follow. Suppose that $d\left(x_{j}, \operatorname{Cen}(\pi)\right)>d\left(x_{j}, \operatorname{Cen}\left(\pi\left[x_{j} \rightarrow\right.\right.\right.$ $w])$. Because $n=3, d\left(x_{j}, C e n(\pi)\right)$ is equal to 1 or 2 . If it is 2 , then without loss of generality $x_{j}=a_{1}$ and $\operatorname{Cen}(\pi)=\left\{a_{3}\right\}$, so $\{\pi\}=\left\{a_{3}\right\}$ and since $C e n$ is unanimous SP1 cannot fail for this $\pi$. If $d\left(x_{j}, \operatorname{Cen}(\pi)\right)=1$, then $x_{j} \in \operatorname{Cen}\left(\pi\left[x_{j} \rightarrow w\right]\right)$. We may assume that $|\pi|>1$, so without loss of generality, $\{\pi\}=\left\{a_{1}, a_{2}\right\},\left\{a_{1}, a_{3}\right\}$, or $\left\{a_{1}, a_{2}, a_{3}\right\}$. Since $x_{j} \notin C e n(\pi)$, in the first case $x_{j}=a_{3}$, and in the second and third cases $x_{j}=a_{1}$ or $a_{3}$, without loss of generality the former. The first case is impossible since $x_{j}$ must be in $\{\pi\}$. In the second and third cases, since $x_{j}=a_{1}$ is in $\operatorname{Cen}\left(\pi\left[x_{j} \rightarrow w\right]\right.$, we cannot have $a_{3}$ in $\left\{\pi\left[x_{j} \rightarrow w\right]\right\}$, which contradicts $\{\pi\}=\left\{a_{1}, a_{3}\right\}$ or $\{\pi\}=\left\{a_{1}, a_{2}, a_{3}\right\}$. We conclude that SP1 holds. 
Suppose $n \geq 4$. By Proposition 5, SP1 and SP2 fail. Next consider $n \geq 5$ and let $\pi=\left(x_{1}, \ldots, x_{k}\right)=\left(a_{1}, a_{1}, \ldots, a_{1}, a_{3}\right)$. Then $\operatorname{Cen}(\pi)=\left\{a_{2}\right\}$. However, $\operatorname{Cen}\left(\pi\left[x_{k} \rightarrow a_{5}\right]\right)=\operatorname{Cen}\left(\left(a_{1}, a_{1}, \ldots, a_{1}, a_{5}\right)\right)=\left\{a_{3}\right\}$, so SP 4 fails.

It is left to prove that SP4 holds for $n=4$. Suppose that $\operatorname{Cen}\left(\pi\left[x_{j} \rightarrow w\right]\right)=\left\{x_{j}\right\}$. Since $w \in\left\{\pi\left[x_{j} \rightarrow w\right]\right\}$ and $w \neq x_{j}$, we have $\left|\pi\left[x_{j} \rightarrow w\right]\right|>1$. Since $\operatorname{Cen}\left(\pi\left[x_{j} \rightarrow w\right]\right)$ has only one element, this eliminates as $\left\{\pi\left[x_{j} \rightarrow w\right]\right\}$ all subsets of $\left\{a_{1}, a_{2}, a_{3}, a_{4}\right\}$ except for the four cases: $\left\{a_{1}, a_{3}\right\},\left\{a_{2}, a_{4}\right\},\left\{a_{1}, a_{2}, a_{3}\right\},\left\{a_{2}, a_{3}, a_{4}\right\}$. By symmetry, we need only consider the first and the third. In both of these cases, $\operatorname{Cen}\left(\pi\left[x_{j} \rightarrow w\right]\right)$ is $\left\{a_{2}\right\}$, which means that $a_{2}=x_{j}$ is also in $\{\pi\}$. Thus, since $\left\{\pi\left[x_{j} \rightarrow w\right]\right\}$ is either $\left\{a_{1}, a_{3}\right\},\left\{a_{1}, a_{2}, a_{3}\right\},\{\pi\}$ is one of $\left\{a_{1}, a_{2}\right\},\left\{a_{2}, a_{3}\right\},\left\{a_{1}, a_{2}, a_{3}\right\}$. In each case $x_{j}=$ $a_{2} \in \operatorname{Cen}(\pi)$, which implies that SP4 holds.

\subsection{Cycles}

We now consider $C e n$ on the cycle $C_{n}$ of $n$ vertices, which we will denote in order on the cycle as $a_{1}, a_{2}, \ldots, a_{n}$. We may consider $n>3$ since $n=3$ gives $K_{3}$ and then, for $k>1$, SP1 through SP4 hold by Proposition 4 .

Proposition 7 For a cycle $C_{n}$ with $n>3$ :

1. Cen satisfies SP1 iff $n=4, k=2 ; n=4, k=3$; or $n=5, k=2$.

2. Cen satisfies SP2 iff $n=4, k=2 ; n=4, k=3$; or $n=5, k=2$.

3. Cen satisfies SP3 iff $n=4, k=2$.

4. Cen satisfies SP4 iff $n=4, k \geq 2 ; n=5, k \geq 2 ; n=6, k \geq 2 ; n=7, k=2 ; n=$ $8, k=2$.

Proof. Note that if $n \geq 6$, then $C_{n}$ has diameter at least 3 , so by Proposition 5, SP1 and SP2 fail. Now let $n=4$ or 5 . Suppose that $k \geq n$. Let $\pi=\left(a_{1}, a_{1}, a_{2}, a_{3}, \ldots, a_{n-1}\right)$. Note that since $n-1 \geq 3, a_{1} \notin \operatorname{Cen}(\pi)$. However, $a_{1} \in \operatorname{Cen}\left(\pi\left[x_{1} \rightarrow a_{n}\right]\right)=V(G)$, and thus condition SP2, and hence also SP1, fails. For SP1 and SP2, this leaves the cases $n=4, k=2 ; n=4, k=3 ; n=5, k=2 ; n=$ $5, k=3 ; n=5, k=4$, which we consider next.

If $n=4$ and $k \leq 3$, then up to symmetry, the only possibilities for $\{\pi\}$ that we need to consider are $\left\{a_{1}\right\},\left\{a_{1}, a_{2}\right\},\left\{a_{1}, a_{3}\right\},\left\{a_{1}, a_{2}, a_{3}\right\}$. In the first case, since $C e n$ is unanimous, SP1 is satisfied and thus so is SP2. In the second case, $\operatorname{Cen}(\pi)=\{\pi\}$ so $d\left(x_{j}, C e n(\pi)\right)=0$ so SP1 and therefore SP2 holds. In the third case, suppose without loss of generality that $j=1$ and that $x_{1}=a_{1}$. Then $d\left(x_{j}, \operatorname{Cen}(\pi)\right)=$ $d\left(x_{j},\left\{a_{2}, a_{4}\right\}\right)=1$. Since $k \leq 3$, the only possibility for $\{\pi\}$ is $\left\{a_{1}, a_{3}\right\}$. It follows that for $w \neq a_{1},\left\{\pi\left[x_{1} \rightarrow w\right]\right\}$ is either $\left\{a_{3}, w\right\}$ or $\left\{a_{1}, a_{3}, w\right\}$, and in each case $a_{1}$ is not in $\operatorname{Cen}\left(\pi\left[x_{1} \rightarrow w\right]\right)$. Hence, SP1 holds and thus so does SP2. In the fourth case, up to interchange of order, $\pi=\left(a_{1}, a_{2}, a_{3}\right)$ since $k \leq 3$. Without loss of generality, 
$j=1$ or $j=2$. Suppose first that $j=1$ and, without loss of generality, $x_{1}=a_{1}$. Then $d\left(x_{1}, \operatorname{Cen}(\pi)\right)=d\left(a_{1}, a_{2}\right)=1$. Then $\left\{\pi\left[x_{1} \rightarrow w\right]\right\}=\left\{a_{2}, a_{3}\right\}$ or $\left\{a_{2}, a_{3}, a_{4}\right\}$ and $a_{1} \notin \operatorname{Cen}\left(\pi\left[x_{1} \rightarrow w\right]\right)$, so $d\left(x_{1}, \operatorname{Cen}\left(\pi\left[x_{1} \rightarrow w\right]\right)\right) \geq 1$. If $j=2$, then $\left\{\pi\left[x_{2} \rightarrow w\right]\right\}=$ $\left\{a_{1}, a_{3}\right\}$ or $\left\{a_{1}, a_{3}, a_{4}\right\}$ and again $a_{1}$ is not in $\operatorname{Cen}\left(\pi\left[x_{2} \rightarrow w\right]\right)$ and $d\left(x_{2}, \operatorname{Cen}\left(\pi\left[x_{2} \rightarrow\right.\right.\right.$ $w])) \geq 1$. This proves SP1 and thus SP2.

Next, let $n=5, k=2$. Then up to symmetry, $\pi=\left(a_{1}, a_{1}\right),\left(a_{1}, a_{2}\right)$, or $\left(a_{1}, a_{3}\right)$ and we may take $x_{1}=a_{1}$. In the first two cases, $d\left(x_{1}, C e n(\pi)\right)=0$ and so SP1 and therefore SP2 holds. In the third case, $\left.d\left(x_{1}, \operatorname{Cen}(\pi)\right)\right)=1$ and $\operatorname{Cen}\left(\pi\left[x_{1} \rightarrow w\right]\right)=$ $\left\{a_{3}\right\},\left\{a_{2}, a_{3}\right\}$ or $\left\{a_{3}, a_{4}\right\}$. In every case, $a_{1} \notin \operatorname{Cen}\left(\pi\left[x_{1} \rightarrow w\right]\right)$ and so $d\left(x_{1}, \operatorname{Cen}\left(\pi\left[x_{1} \rightarrow\right.\right.\right.$ $w]) \geq 1$, which gives SP1 and thus SP2.

To complete the proof for SP1 and SP2, there are two more cases. First, let $n=5, k=3$. Take $\pi=\left(a_{1}, a_{1}, a_{3}\right)$. Then $x_{1}=a_{1} \notin \operatorname{Cen}(\pi)$ but $x_{1}=a_{1} \in$ $\operatorname{Cen}\left(\pi\left[x_{1} \rightarrow a_{5}\right]\right)=\operatorname{Cen}\left(a_{5}, a_{1}, a_{3}\right)=V(G)$. Thus, SP2 fails and, therefore, SP1 fails. Next, let $n=5, k=4$. Take $\pi=\left(a_{1}, a_{1}, a_{1}, a_{3}\right)$. Then $x_{1}=a_{1} \notin C e n(\pi)$ but $x_{1} \in \operatorname{Cen}\left(\pi\left[x_{1} \rightarrow a_{5}\right]\right)=V(G)$, so SP2 fails and therefore so does SP1.

Now consider SP3. Let $n \geq 4, k \geq 3$. Take $\pi=\left(a_{1}, a_{1}, \ldots, a_{1}, a_{2}\right)$. Then $\operatorname{Cen}(\pi)=$ $\left\{a_{1}, a_{2}\right\}$ but $\operatorname{Cen}\left(\pi\left[x_{1} \rightarrow a_{n}\right]\right)=\left\{a_{1}\right\}$, which shows that SP3 fails. Now let $n \geq 5, k=$ 2. Let $\pi=\left(a_{1}, a_{n}\right)$. Then $\operatorname{Cen}(\pi)=\left\{a_{1}, a_{n}\right\}$ but $\operatorname{Cen}\left(\pi\left[x_{1} \rightarrow a_{2}\right]\right)=\left\{a_{1}\right\}$, so SP3 fails. Finally, if $n=4, k=2$, suppose $\operatorname{Cen}\left(\pi\left[x_{1} \rightarrow w\right]\right)$ has only one element, $a_{1}$. Since $n=4, k=2$, we must have $\pi=\left(a_{1}, a_{1}\right)$, which is impossible since $w \neq x_{1}$. Thus, SP3 holds.

Finally, consider SP4. First, suppose $n=4$. If $\operatorname{Cen}\left(\pi\left[x_{j} \rightarrow w\right]\right)$ has one element $x_{j}$, then without loss of generality $\left\{\pi\left[x_{j} \rightarrow w\right]\right\}=\left\{a_{1}\right\}$ or $\left\{a_{1}, a_{2}, a_{3}\right\}$. The former case is impossible since $x_{j}=a_{1}$ and $w \neq x_{j}$ must both be in $\left\{\pi\left[x_{j} \rightarrow w\right]\right\}$. In the latter case, $\operatorname{Cen}\left(\pi\left[x_{j} \rightarrow w\right]\right)=\left\{a_{2}\right\}$ and $\{\pi\}=\left\{a_{1}, a_{2}\right\},\left\{a_{2}, a_{3}\right\},\left\{a_{1}, a_{3}\right\}$, or $\left\{a_{1}, a_{2}, a_{3}\right\}$. In each case, $a_{2} \in \operatorname{Cen}(\pi)$, which implies that SP 4 holds.

Next, let $n=5$. If $\operatorname{Cen}\left(\pi\left[x_{j} \rightarrow w\right]\right)$ has one element $x_{j}$, then without loss of generality $\left\{\pi\left[x_{j} \rightarrow w\right]\right\}=\left\{a_{1}\right\},\left\{a_{1}, a_{3}\right\}$ or $\left\{a_{1}, a_{2}, a_{3}\right\}$. The former case is impossible as with $n=4$. In the other two cases, $\left\{a_{2}\right\}=\operatorname{Cen}\left(\pi\left[x_{j} \rightarrow w\right]\right)$ and $x_{j}=a_{2}$. If $\left\{\pi\left[x_{j} \rightarrow w\right]\right\}=\left\{a_{1}, a_{3}\right\}$, then $\{\pi\}=\left\{a_{1}, a_{2}\right\},\left\{a_{2}, a_{3}\right\}$, or $\left\{a_{1}, a_{2}, a_{3}\right\}$. In each case, $a_{2} \in \operatorname{Cen}(\pi)$, which implies that SP4 holds. If $\left\{\pi\left[x_{j} \rightarrow w\right]\right\}=\left\{a_{1}, a_{2}, a_{3}\right\}$, then we have the same possible sets $\{\pi\}$ and again we get SP4.

Suppose $n=6$. If $\operatorname{Cen}\left(\pi\left[x_{j} \rightarrow w\right]\right)$ has one element $x_{j}$, then without loss of generality $\left\{\pi\left[x_{j} \rightarrow w\right]\right\}=\left\{a_{1}\right\},\left\{a_{1}, a_{3}\right\},\left\{a_{1}, a_{2}, a_{3}\right\}$, or $\left\{a_{1}, a_{2}, a_{3}, a_{4}, a_{5}\right\}$. The first three cases are handled as for $n=5$. In the fourth case, $\operatorname{Cen}\left(\pi\left[x_{j} \rightarrow w\right]\right)=\left\{a_{3}\right\}$. Now $\{\pi\}$ has to be one of the sets $\left\{a_{1}, a_{2}, a_{3}, a_{4}, a_{5}\right\},\left\{a_{1}, a_{2}, a_{3}, a_{4}\right\},\left\{a_{1}, a_{2}, a_{3}, a_{5}\right\}$, $\left\{a_{1}, a_{3}, a_{4}, a_{5}\right\},\left\{a_{2}, a_{3}, a_{4}, a_{5}\right\}$. Since $a_{3} \in C e n(\pi)$ in all cases, SP4 holds.

Next, take $n=7$. When $k \geq 4$, consider $\pi=\left(a_{1}, a_{1}, \ldots, a_{1}, a_{2}, a_{3}\right)$. Then $\operatorname{Cen}(\pi)=$ $\left\{a_{2}\right\}$. Now $\operatorname{Cen}\left(\pi\left[x_{k-2} \rightarrow a_{6}\right]\right)=\operatorname{Cen}\left(\left(a_{1}, a_{1}, \ldots, a_{1}, a_{6}, a_{2}, a_{3}\right)\right)=\left\{a_{1}\right\}$, so SP 4 fails. (Note that $k \geq 4$ is used since it implies that $k-2 \geq 2$ and thus $\{\pi\}$ has $a_{1}$ in it.) When $k=3$, consider $\pi=\left(a_{1}, a_{2}, a_{3}\right)$, with $\operatorname{Cen}(\pi)=\left\{a_{2}\right\}$. Then $\operatorname{Cen}\left(\pi\left[x_{3} \rightarrow a_{5}\right]\right)=\operatorname{Cen}\left(\left(a_{1}, a_{2}, a_{5}\right)\right)=\left\{a_{3}\right\}$, so SP4 fails. Suppose next that $k=2$ and that $\left\{\pi\left[x_{j} \rightarrow w\right]\right\}=\left\{x_{j}\right\}$. Since $k=2$, without loss of generality 
$\pi\left[x_{j} \rightarrow w\right]=\left(a_{1}, a_{1}\right)$ or $\left(a_{1}, a_{3}\right)$. In the former case, $x_{j}=a_{1}$ is in $\operatorname{Cen}(\pi)$. In the latter case, $x_{j}=a_{2}$ and $\{\pi\}=\left\{a_{1}, a_{2}\right\}$ or $\left\{a_{2}, a_{3}\right\}$, so $x_{j} \in C e n(\pi)$ and SP 4 holds.

To handle the case $n=8$, suppose first that $k \geq 4$, and consider $\pi=\left(a_{1}, a_{1}, \ldots, a_{1}, a_{2}, a_{3}\right)$. (As in the case $n=7$, the assumption $k \geq 4$ is used.) Then $C e n(\pi)=\left\{a_{2}\right\}$. Now $\operatorname{Cen}\left(\pi\left[x_{k} \rightarrow a_{5}\right]\right)=\operatorname{Cen}\left(\left(a_{1}, a_{1}, \ldots, a_{1}, a_{2}, a_{5}\right)\right)=\left\{a_{3}\right\}$, so SP4 fails. When $k=3$, the same example as with $n=7$ shows that SP4 fails. Finally, take $k=2$. That SP4 holds follows in the same way as with $n=7$.

To conclude the proof, consider $n \geq 9$. Take $\pi=\left(a_{1}, a_{1}, \ldots, a_{1}, a_{3}\right)$. Note that $\operatorname{Cen}(\pi)=\left\{a_{2}\right\}$, but $\operatorname{Cen}\left(\pi\left[x_{k} \rightarrow a_{5}\right]\right)=\operatorname{Cen}\left(\left(a_{1}, a_{1}, \ldots, a_{1}, a_{5}\right\}\right)=\left\{a_{3}\right\}$, so SP 4 fails.

\section{The median function on median graphs}

We now study how the median function behaves on median graphs with respect to strategy-proofness. Median graphs form a class of bipartite graphs that include trees and $n$-cubes. Specifically, a median graph is a connected graph $G=(V, E)$ such that for every three vertices $x, y, z \in V$, there is a unique vertex $w$ on a shortest-length path between each pair of $x, y, z$. Let $I(x, y)=\{w \in V: d(x, w)+d(w, y)=d(x, y)\}$. Then it is easy to see that $G$ is a median graph if and only if $|I(x, y) \cap I(x, z) \cap I(y, z)|=1$ for all $x, y, z \in V$.

First we present some necessary concepts and results for arbitrary graphs. Then we concentrate on median graphs and recapitulate some necessary notation and results from $[5,9,10]$.

Let $G=(V, E)$ be a connected graph. A subgraph $H$ of $G$ is convex if, for any two vertices $x$ and $y$ of $H$, all shortest $x, y$-paths lie completely in $H$. Note that convex subgraphs are induced. A subset $W$ of $V$ is convex if it induces a convex subgraph. A subgraph $H$ is gated if, for any vertex $w$ there exists a unique vertex $x$ in $H$ such that for each vertex $y$ of $H$ there exists a shortest $w, y$-path through $x$. This vertex $x$ is the gate for $w$ in $H$. Clearly, if $H$ is gated, then the gate for $w$ in $H$ is the vertex of $H$ closest to $w$. It is also the unique vertex $z$ in $H$ such that any shortest $w, z$-path intersects $H$ only in $w$. A gated subset of vertices is a subset that induces a gated subgraph. Note that gated subgraphs are convex, but the converse need not be the case. A simple consequence of the theory on median graphs is that convex sets in a median graph are always gated. Let $\pi$ be a profile on $G$ and $u v \in E$. By $W_{u v}$ we denote the subset of $V$ of all vertices closer to $u$ than to $v$, by $G_{u v}$ the subgraph induced by $W_{u v}$. The subgraphs $G_{u v}, G_{v u}$ form a so-called split: the sets $W_{u v}, W_{v u}$ are disjoint with $V$ as their union. We call $G_{u v}$ and $G_{v u}$ split-sides. Split-sides are convex subgraphs, and hence gated.

Let $\pi$ be a profile, $\pi_{u v}$ be the subprofile of $\pi$ consisting of the vertices in $\pi$ closer to $u$ than $v$, and let $l\left(\pi_{u v}\right)$ denote the number of terms in the sequence $\pi_{u v}$. Theorem 3 of [5] tells us that, for any profile $\pi$ and any edge $u v$ with $l\left(\pi_{u v}\right)>l\left(\pi_{v u}\right)$ we have 
$\operatorname{Med}(\pi) \subseteq G_{u v}$. An important consequence of this theorem is that

$$
\operatorname{Med}(\pi)=\bigcap_{l\left(\pi_{u v}\right)>l\left(\pi_{v u}\right)} G_{u v}
$$

Since the intersection of convex subgraphs is again convex, median sets of profiles are thus convex, and hence also gated.

For any two vertices $u, v$ in $G$ the set of neighbors of $u$ in $I(u, v)$ is denoted by $N_{1}(u, v)$. Loosely speaking these are precisely the vertices that are one step closer to $v$ from $u$. Let $G_{x / v}=\bigcap_{u \in N_{1}(v, x)} G_{v u}$, which signifies all vertices that are "behind" $v$ seen from $x$, that is, all vertices that can be reached from $x$ by a shortest path passing through $v$.

Lemma 8 Let $x$ and $v$ be vertices in a median graph $G$. Then $v$ is the gate for $x$ in $\bigcap_{u \in N_{1}(v, x)} G_{v u}$.

Proof. Since split-sides are convex, the subgraph $G_{x / v}=\bigcap_{u \in N_{1}(v, x)} G_{v u}$ is convex and hence gated. By definition, any shortest $x, v$-path intersects $G_{x / v}$ only in $v$. So indeed $v$ is the gate for $x$ in this subgraph.

Corollary 9 Let $\pi=\left(x_{1}, x_{2}, \ldots, x_{k}\right)$ be a profile on a median graph $G$. If $x_{j}$ is not in $\operatorname{Med}(\pi)$, and $m$ is the gate of $x_{j}$ in $\operatorname{Med}(\pi)$, then $\operatorname{Med}\left(\pi\left[x_{j} \rightarrow w\right]\right)$ is contained in $G_{x_{j} / m}$.

Proof. First we show that $\operatorname{Med}(\pi)$ lies in $G_{x_{j} / m}$. Let $u$ be any neighbor of $m$ in $I\left(x_{j}, m\right)$. Then $u$ is not in $\operatorname{Med}(\pi)$, so a majority of $\pi$ lies in $G_{m u}$, whence $\operatorname{Med}(\pi)$ lies in $G_{m u}$, and we are done.

Now we replace $x_{j}$ by $w$, thus obtaining the profile $\rho=\pi\left[x_{j} \rightarrow w\right]$. Take a neighbor $u$ of $m$ in $I(x, m)$. Note that a majority of $\pi$ lies in $G_{m u}$ and a minority lies in $G_{u m}$, and $x_{j}$ belongs to this minority. So, no matter where $w$ is located, a majority of $\rho$ still lies in $G_{m u}$. Hence $\operatorname{Med}(\rho)$ is contained in $G_{m u}$. This settles the proof.

Theorem 10 Let $G$ be a median graph. Then Med $: V^{k} \rightarrow 2^{V} \backslash\{\emptyset\}$ satisfies $S P 1$ (and therefore SP2 and SP4) for any $k$.

Proof. Let $\pi=\left(x_{1}, x_{2}, \ldots, x_{k}\right)$ be a profile on $G$ such that $x_{j}$ is not in $\operatorname{Med}(\pi)$, and let $w$ be any vertex of $G$. Let $m$ be the gate of $x_{j}$ in $\operatorname{Med}(\pi)$. Note that in $G, d\left(x_{j}, \operatorname{Med}(\pi)\right)=d\left(x_{j}, m\right)$. By Corollary $9, \operatorname{Med}\left(\pi\left[x_{j} \rightarrow w\right]\right)$ lies in $G_{x_{j} / m}$. So each vertex $y$ in $\operatorname{Med}\left(\pi\left[x_{j} \rightarrow w\right]\right)$ can be reached from $x_{j}$ via a shortest path passing through $m$. Hence $d\left(x_{j}, m\right) \leq d\left(x_{j}, y\right)$ for all $y \in\left\{\pi\left[x_{j} \rightarrow w\right]\right\}$, and we are done. 


\section{Conclusions and Future Work}

This note has introduced four notions of strategy-proofness and illustrated them for several location functions and for several types of graphs. We have only begun to investigate this subject and, even for this relatively small beginning, have left open questions to be addressed.

For instance, we have given an example of a function (the average function) that is an isotone, onto location function but does not satisfy SP1. We believe that under certain conditions, the converse holds, but leave the investigation of such conditions to future work.

Proposition 5 shows that for every graph of diameter at least 3 , when $k>1$, Cen violates SP1 and SP2. We have left open the question of whether this is also true of SP3 and SP4.

Section 5 shows that SP1, and therefore SP2 and SP4, hold for median graphs. It leaves open this question for SP3.

Section 4 determines the cases where SP1 through SP4 hold for the center function on paths and cycles. For the median function, since a path is a median graph, Section 5 handles SP1, SP2, and SP4. SP3 remains open. We have not attempted to categorize when these conditions of strategy-proofness hold for cycles. For trees, the fact that they are median graphs shows that SP1, SP2, and SP4 hold for the median function. SP3 remains open. For the center function, the case of trees other than paths remains an area for future research.

\section{References}

[1] N. Alon, M. Feldman, A.D. Procaccia and M. Tennenholtz, Strategyproof approximation of the minimax on networks, Math. of Operations Research 35(2010) $513-526$.

[2] V.I. Danilov, The structure of non-manipulable social choice rules on a tree, Math. Soc. Sci. 27 (1994) 123 - 131.

[3] S. Klavžar and H.M. Mulder, Median graphs: characterizations, location theory, and related structures, J. Combin. Math. Combin. Comput. 30 (1999) 103-127.

[4] F.R. McMorris, H.M. Mulder and R.C. Powers, The median function on distributive semilattices, Discrete Appl. Math. 127 (2003) 319 - 324.

[5] F.R. McMorris, H.M. Mulder and F.S. Roberts, The median procedure on median graphs, Discrete Appl. Math. 84 (1998) 165 - 181.

[6] F.R. McMorris, F.S. Roberts and C. Wang, The center function on trees, Networks 38 (2001) $84-87$. 
[7] E. Miyagawa, Locating libraries on a street, Soc. Choice Welfare 18 (2001) 527 $-541$.

[8] H. Moulin, On strategy proofness and single peakedness, Public Choice 35 (1980) $437-455$.

[9] H.M. Mulder, The structure of median graphs, Discrete Math. 24 (1978) 197 204.

[10] H.M. Mulder, The Interval Function of a Graph, Mathematical Centre Tracts 132, Mathematisch Centrum, Amsterdam, 1980.

[11] M.R. Sanver, Strategy-proofness of the plurality rule over restricted domains, Econ. Theory 39 (2009) $461-471$.

[12] J. Schummer and R.V. Vohra, Strategy-proof location on a network, J. Econ. Theory 104 (2002) $405-428$.

[13] A.D. Taylor, Social Choice and the Mathematics of Manipulation, Cambridge University Press, 2005. 